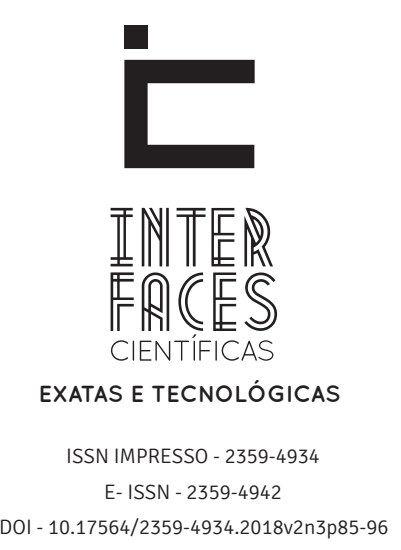

\title{
PRODUÇÃO CIENTÍFICA SOBRE TECNOLOGIA DA INFORMAÇÃO VERDE: ESTUDO A PARTIR DE PERIÓDICOS NACIONAIS
}

\author{
SCIENTIFIC PRODUCTION ON GREEN INFORMATION TECHNOLOGY: STUDY FROM NATIONAL JOURNALS
}

PRODUCCIÓN CIENTÍFICA SOBRE TECNOLOGÍA DE LA INFORMACIÓN VERDE: ESTUDIO A PARTIR DE PERIÓDICOS NACIONALES

Antonio Karlos Valença ${ }^{1}$

Antonio Eduardo Albuquerque Junior ${ }^{3}$
Rodrigo César Reis de Oliveira²

Vivian Farias Melo ${ }^{4}$

\section{RESUMO}

É evidente o crescimento do tema sustentabilidade ambiental em nosso cotidiano, não sendo de se estranhar que este tema seja vivenciado também no ambiente empresarial. Com isso tem-se uma busca crescente do mercado para seguir esta tendência, fato que acontece também com a tecnologia da informação. Dessa forma, o presente artigo teve como objetivo analisar a produção científica nacional relativa às práticas da Tecnologia da Informação Verde (Green IT) nas organizações, sob a perspectiva de que é necessário entender o contexto no qual ela está inserida e para que seja possível o seu gerenciamento de modo sustentável. Para atender este objetivo, foram analisados artigos científicos submetidos a periódicos nacionais, sob o método de revisão bibliográfica no período de 2009-2016. Após este estudo foi possível concluir que alguns fatores como altos custos e cumprimento da legislação são primordiais quando se decide adotar essa tendência. Vale ressaltar que é necessária que haja o devido conhecimento do que seja TI Verde e quais os fatores que influenciam positivamente na não adoção dessas práticas.

\section{PALAVRAS-CHAVE}

Produção Científica, TI Verde, Sustentabilidade. 


\section{ABSTRACT}

It is evident the growth of the theme environmental sustainability in our daily life, not surprising that this theme is also experienced in the business environment. With this there is an increasing search of the market to follow this tendency, fact that also happens with the technology of the information. Thus, the present article aimed to analyze the national scientific production related to Green IT practices in organizations, under the perspective that it is necessary to understand the context in which it is inserted and to make possible the management in a sustainable way. In order to meet this objective, scientific articles submitted to national journals were ana-

\section{RESUMEN}

Es evidente el crecimiento del tema sustentabilidad ambiental en nuestro cotidiano, no siendo de extrañarse que este tema sea vivenciado también en el ambiente empresarial. Con eso se tiene una búsqueda creciente del mercado para seguir esta tendencia, hecho que ocurre también con la tecnología de la información. De esta forma, el presente artículo tuvo como objetivo analizar la producción científica nacional relativa a las prácticas de la Tecnología de la Información Verde (Green IT) en las organizaciones, desde la perspectiva de que es necesario entender el contexto en el que está inserta y para que sea posible su gestión de manera sostenible. Para atender este objetivo, fueron analizados artículos científicos sometidos lyzed under the method of bibliographical review in the period 2009-2016. After this study it was possible to conclude that some factors such as high costs and compliance with legislation are paramount when deciding to adopt this trend. It is worth mentioning that it is necessary to have the proper knowledge of what Green IT is and what factors influence positively in the non-adoption of these practices.

\section{KEYWORDS}

Scientific Production, Green IT, Sustainability.

a periódicos nacionales, bajo el método de revisión bibliográfica en el período 2009-2016. Después de este estudio fue posible concluir que algunos factores como altos costos y cumplimiento de la legislación son primordiales cuando se decide adoptar esa tendencia. Es importante resaltar que es necesario que haya el debido conocimiento de lo que sea TI Verde y cuáles son los factores que influyen positivamente en la no adopción de esas prácticas.

\section{PALABRAS CLAVE}

Producción Científica, TI Verde, Sostenibilidad. 


\section{INTRODUÇ̄̃O}

As organizações têm experimentado uma constante busca por soluções tecnológicas inovadoras e criativas, que têm sido incorporadas aos seus processos internos (KUBOTA; DA ROSA, 2011). A necessidade de oferecer novos produtos e serviços e a constante busca por inovação tecnológica, tendo em vista a sobrevivência no mercado e o lucro, adquiriram relevância evidente para as organizações e, muitas vezes, questões referentes à sustentabilidade são deixadas de lado (MARCONDES; BACARJI, 2010; DANIEL; AGUIAR, 2014).

Por outro lado, tem havido um crescimento da preocupação com os impactos das ações humanas no meio ambiente, o que inclui a geração e descarte de resíduos, o que é inerente a qualquer atividade (PINHEIRO et al., 2016). Diante do consumo excessivo de bens e serviços, muitas empresas têm se empenhado no desenvolvimento de projetos e ações socioambientais, buscando ser transparentes perante a sociedade e aos órgãos legais (PUPPIN, 2005; SOUZA; BRIGHENTI; HEIN, 2016).

Bozzelli, Gu e Lago (2013) citam alguns exemplos, como a redução do consumo de energia e emissões de carbono, tendo como o objetivo alcançar melhores níveis de sustentabilidade. Diante desse contexto, a busca por processos e produtos sustentáveis também representa desafios que a comunidade da Tecnologia da Informação (TI) está enfrentando para lidar com questões ambientais geradas por sistemas de TI.

Para Hepper, Hansen e Santos (2016), as iniciativas sustentáveis podem ser consideradas como uma necessidade, isto tem levado organizações a buscarem o desenvolvimento sustentável, tanto por reconhecerem tal necessidade quanto por buscarem atender a um público crescente de consumidores cada vez mais conscientes, dentro das exigências legais. Essa tendência pela busca da sustentabilidade tem sido evidenciada também nas práticas de TI, reconhecida como TI Verde.

A partir dessa nova modalidade da Tecnologia da Informação, o presente artigo tem o objetivo de analisar a produção científica brasileira sobre a adoção das práticas da Tecnologia da Informação Verde (Green IT) nas organizações, sob a perspectiva de que é necessário entender o contexto no qual ela está inserida e para que seja possível o seu gerenciamento de modo sustentável.

Como justificativa, sabe-se que a Tecnologia da Informação (TI) contribui positivamente para a redução dos custos, bem como com um aumento dos ganhos em produtividade, prospecção de novos mercados, facilidade de relacionamento com clientes e fornecedores, além de possibilitar um melhor conhecimento do mercado no qual se atua (OLIVEIRA et al., 2013). Porém, segundo Melville (2010), o uso ineficiente da TI, além de causar danos ao meio ambiente, pode resultar em maiores custos para as organizações, podendo, de fato, fazê-las perder vantagens competitivas. Para Salles e outros autores (2013), colocar em prática a chamada TI Verde exige algumas mudanças de comportamento e tecnologias propriamente ditas, além de investimentos financeiros que viabilizem o processo de adoção dessas práticas.

\section{SUSTENTABILIDADE E A TECNOLOGIA DA INFORMAÇÃO VERDE}

O conceito de sustentabilidade pode ser compreendido como a ligação entre os recursos naturais e a valorização do ser humano, de modo que se torne sociável e capaz de desenvolver-se sem agredir o meio em que vive e de suprir suas necessidades sem comprometer as gerações futuras (DANIEL, 2014). Para tanto, a análise da sustentabilidade direcionada ao aspecto ambiental não pode ser mais realizada separadamente dos aspectos sociais e econômicos (HEPPER; HANSEN; SANTOS, 2016).

A adoção de práticas sustentáveis pode levar a organização a vislumbrar-se de modo imperativo, podendo gerar vantagens competitivas de mercado e aumentar o sucesso das empresas, o que pode se caracterizar como uma estratégia de diferenciação (WAGNER; SCHALTEGGER, 2003; MORAIS; OLIVEIRA; SOUZA, 2014). As práticas sustentáveis de uma organização são norteadas principalmente pela gestão dos recursos intrínsecos ao seu core business, cujo uso racional impacta positivamente nos resultados 
econômicos, sociais e ambientais da empresa, contribuindo para seu posicionamento como organização sustentável (BUENAFUENTE et al., 2010).

Peçanha e lizuka (2014) comentam que as questões ambientais, sociais e econômicas têm tido uma relevância muito grande nos últimos anos, sendo pauta de diversos fóruns, encontros e seminários acadêmicos e profissionais, integrando o processo de tomada de decisão e planejamento estratégico para as organizações e sociedade. Esses três aspectos foram abordados por Elkington (1994) em seus estudos como sendo o Triple Bottom Line ou Tripé da Sustentabilidade, que consiste em integrar os cenários ambiental, econômico e social de modo que sejam considerados para o equilíbrio da organização e devem dar suporte ao desenvolvimento e à execução de ações sustentáveis.

Figura 1 - Triple Bottom Line

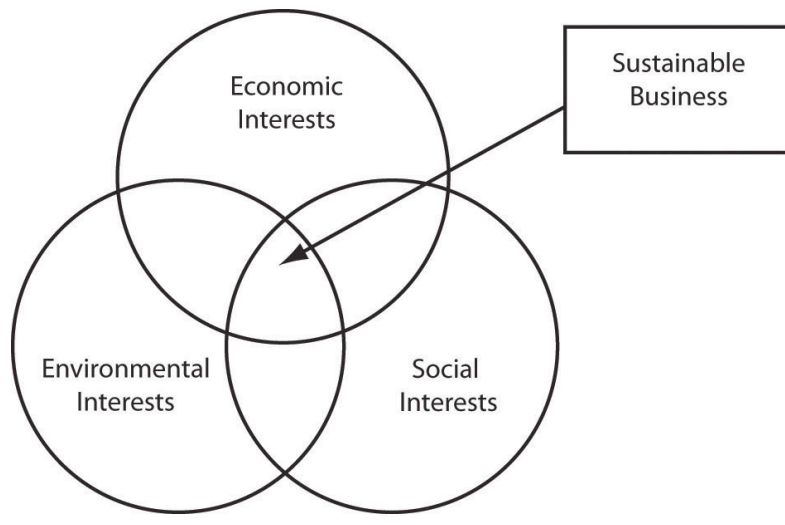

Fonte: Adaptado de Elkington (1994).

Ao considerar a Figura 1, é importante ressaltar que o aspecto econômico não engloba apenas a economia formal, mas também envolve atividades informais que disponibilizam serviços, que são obtidos a partir do feedback, de modo que auxilia a tomada de decisão de caráter financeiro, sustentando o crescimento econômico da organização. 0 aspecto ambiental, por sua vez, estimula que as organizações considerem o resultado de suas atividades sobre meio ambiente e formatem ações que contribuam para a melhoria de tal questão, respeitando a capacidade de suporte dos ecossistemas. Já aspecto social está relacionado à qualidade dos seres humanos e de suas relações, considerando suas habilidades, dedicação e experiências, de modo a desenvolver estratégias para a melhoria do seu bem-estar (ALMEIDA, 2002; ARAÚJO; INOMATA; RADOS, 2014; SEIFFERT, 2011).

Essas práticas sustentáveis, alinhadas com as tecnologias da informação, constituem a chamada TI Verde. É um movimento recente, porém está se tornando algo proeminente, apesar de não apresentar um conceito inteiramente definido e tão pouco representa um conjunto predeterminado de práticas aceitas e aplicadas ou padronizadas em todas as organizações (LUNARDI; FRIO; BRUM, 2011; CHONG; OLESEN, 2017).

Neste sentido, Murugesan (2010) conceitua que a Tecnologia de Informação Verde pode ser considerada como aquela que se utiliza de estudos para projetar, fabricar, utilizar e descartar produtos e subprodutos computacionais, pressupondo efetividade para garantir níveis mínimos de impactos negativos ao meio ambiente. Além disso, está atenta ao desperdício, à economia, à eficiência energética e ao custo total de propriedade, englobando o custo de descarte e reciclagem e compreende, ainda, instrumentos para controlar, orientar e comunicar as práticas usadas ( $\mathrm{SCH}-$ MIDT et al., 2010).

Para potencializar seu uso, Watson, Boudreau e Chen (2010) pautam três objetivos que tornam as práticas da TI Verde substanciais: Ecoeficiência, Ecoequidade e Ecoeficácia.

A ecoeficiência pode ser entendida como sendo a entrega de produtos e serviços com preços diferenciados que satisfaçam as necessidades dos clientes melhorando a qualidade de vida, enquanto reduzem os impactos ecológicos e a intensidade de recursos utilizados em todo o ciclo de vida (DESIMONE; POPOFF, 1997).

A ecoequidade, na visão de Gray e Bebbington (2000), se refere à equidade entre as pessoas e as gerações, e, em particular, à igualdade dos direitos de todas as pessoas aos recursos ambientais. Lunardi, Frio e Brum (2011) comentam que, para aumentar a 
equidade nas empresas, é necessário potencializar a influência de mudar as normas sociais do comportamento do consumidor e as normas corporativas no comportamento organizacional.

Por fim, a ecoeficácia é considerada como a última solução dos problemas ecológicos, pois requer uma mudança de mentalidade e transformação dos negócios, pois as pessoas precisam parar de danificar o meio ambiente e destruir os recursos não renováveis (LUNARDI;
FRIO; BRUM, 2011). Na visão de McDonough e Braungart (2002), a ecoeficácia permeia o trabalhar nas coisas certas, ou seja, trabalhar nos produtos, serviços e sistemas que tragam resultados consistentes para a organização, ao invés do foco restrito nos processos internos.

No estudo feito por Lunardi, Frio e Brum (2011), foram identificadas 37 diferentes práticas de TI Verde, adotadas pelas organizações, sendo estas classificadas em sete categorias gerais, conforme Quadro 1.

Quadro 1 - Práticas de TI Verde

\begin{tabular}{|c|c|}
\hline Práticas de TI Verde & Descrição \\
\hline $\begin{array}{l}\text { Práticas de conscien- } \\
\text { tização }\end{array}$ & $\begin{array}{l}\text { Campanhas internas focadas no impacto ambiental, escolha de fornecedores verdes, a } \\
\text { presença de uma política de sustentabilidade, o teletrabalho, a construção de prédios } \\
\text { verdes, a análise da eficiência energética dos equipamentos eletroeletrônicos e a pre- } \\
\text { sença de comitês de sustentabilidade. }\end{array}$ \\
\hline Datacenter verde & $\begin{array}{l}\text { As práticas verdes direcionadas aos datacenters dizem respeito, especialmente, à virtu- } \\
\text { alização, seja pela consolidação de desktops ou, ainda, dos servidores. Datacenters mais } \\
\text { verdes não só economizam energia, como também reduzem a necessidade de expansão } \\
\text { de infraestrutura para lidar com a demanda crescente por energia e mais resfriamento. }\end{array}$ \\
\hline $\begin{array}{l}\text { Descarte e recicla- } \\
\text { gem }\end{array}$ & $\begin{array}{l}\text { Mostram um maior comprometimento das empresas quanto à utilização, reciclagem e } \\
\text { descarte dos recursos naturais não-renováveis. }\end{array}$ \\
\hline $\begin{array}{l}\text { Fontes alternativas } \\
\text { de energia }\end{array}$ & $\begin{array}{l}\text { A geração de energia alternativa passa pelo uso de biodiesel, combustíveis não-fósseis, } \\
\text { vento e até mesmo as ondas do mar - todas, energias renováveis. }\end{array}$ \\
\hline Hardware & $\begin{array}{l}\text { O desenvolvimento de equipamentos mais eficientes e econômicos energeticamente, a } \\
\text { substituição de monitores CRT por LCD, a eliminação de componentes nocivos nos pro- } \\
\text { dutos, o uso de componentes reciclados na confecção de produtos novos e o aumento do } \\
\text { ciclo de vida dos produtos são algumas dessas práticas. }\end{array}$ \\
\hline Software & $\begin{array}{l}\text { Pode ser visto sob a ótica de tornarem os programas computacionais mais eficientes } \\
\text { (consumindo menos energia, otimizando seu processamento e desabilitando funções ou } \\
\text { dispositivos quando não utilizados) ou, ainda, para gerenciar o consumo de energia de } \\
\text { acordo com a utilização do equipamento pelas empresas, controlar a emissão de gases } \\
\text { ou a qualidade da água e projetar novos produtos mais eficientes. }\end{array}$ \\
\hline Impressão & $\begin{array}{l}\text { A maior preocupação ainda é o uso do papel. Mas com a realização de algumas medidas, } \\
\text { as organizações podem reduzir o impacto ambiental das impressoras e das impressões. } \\
\text { As práticas utilizadas têm sido o monitoramento das impressões, a digitalização de do- } \\
\text { cumentos, a terceirização das impressões, a impressão frente-e-verso, a consolidação de } \\
\text { impressoras, o uso de papel reciclado e o uso de multifuncionais. }\end{array}$ \\
\hline
\end{tabular}

Fonte: Lunardi, Frio e Brum (2011). 
Ainda segundo a pesquisa, algumas dessas práticas exigem elevados investimentos (especialmente aquelas ligadas aos datacenters, às fontes alternativas de energia e à substituição de equipamentos obsoletos por novos), boa parte das práticas de sustentabilidade pode ser adotada sem que a saúde financeira da empresa seja comprometida, apenas dependendo do esforço e da vontade dos usuários e do apoio e direcionamento da organização.

\section{PROCEDIMENTOS METODOLÓGICOS}

0 presente trabalho teve como objetivo realizar uma revisão de publicações científicas no Brasil, sobre o que já foi estudado a respeito do tema Adoção da Tecnologia da Informação Verde. Desse modo, foi realizada uma revisão bibliográfica que consiste em um estudo, com base em trabalhos já publicados em livros, artigos, revistas, periódicos científicos, teses, dissertações e anais de eventos científicos (GIL, 1999).

No caso deste trabalho, buscou-se identificar artigos científicos publicados em periódicos nacionais e selecionados de acordo com a classificação Qualis, disponibilizada na plataforma Sucupira, da Coordenação de Aperfeiçoamento de Pessoal de Nível Superior (CAPES). A pesquisa nos periódicos foi baseada nas seguintes palavras-chave: Green IT, TI Verde e Tecnologia da Informação Verde. Após o exame do material, foram selecionados os trabalhos publicados em revistas científicas com Qualis A1, A2, B1, B2 e B3 classificados no Quadriênio 2013-2016, tendo como período de publicação das obras 2009 a 2016.

\section{APRESENTAÇ̃̃O E DISCUSSÃO DOS RESULTADOS}

No Quadro 2 são apresentados os autores, ano, o periódico no qual foi publicado os artigos, seguido dos seus devidos títulos. Abaixo do Quadro são apresentados os objetivos dos estudos a descrição das propostas dos autores, bem como as conclusões a que chegaram com os estudos que tiveram como pauta a adoção de Tecnologias da Informação Verde nas organizações.

Quadro 2 - Principais trabalhos sobre o tema

\begin{tabular}{|l|l|l|}
\hline AUTORES/ANO & PERIÓDICO & TíTUTO DO TRABALHO \\
\hline $\begin{array}{l}\text { Radons, Battistella e } \\
\text { Grohmann (2012) }\end{array}$ & $\begin{array}{l}\text { Revista Brasileira de } \\
\text { Administração Científica }\end{array}$ & $\begin{array}{l}\text { Vantagem competitiva das empresas: qual a } \\
\text { influência da inovação verde? }\end{array}$ \\
\hline $\begin{array}{l}\text { Lunardi, Lerch e Salles } \\
\text { (2014) }\end{array}$ & Revista de Administração & $\begin{array}{l}\text { Desenvolvimento de uma escala para } \\
\text { avaliar o grau de utilização da tecnologia da } \\
\text { informação verde pelas organizações }\end{array}$ \\
\hline Salles et al. (2016) & $\begin{array}{l}\text { Revista de Administração } \\
\text { Contemporânea }\end{array}$ & $\begin{array}{l}\text { Tecnologia da Informação Verde: Um Estudo } \\
\text { sobre sua Adoção nas Organizaçães }\end{array}$ \\
\hline Dolci et al. (2015) & $\begin{array}{l}\text { Revista de Administração de } \\
\text { Empresas }\end{array}$ & $\begin{array}{l}\text { Implementation of Green IT in Organizations: } \\
\text { a structurational view }\end{array}$ \\
\hline Castro et al. (2016) & $\begin{array}{l}\text { Revista de Gestão Ambiental e } \\
\text { Sustentabilidade - GeAS }\end{array}$ & $\begin{array}{l}\text { O Marketing Verde na Tecnologia da } \\
\text { Informação: percepções das atitudes e } \\
\text { comportamentos dos profissionais de TI e } \\
\text { ações para incrementar o marketing verde } \\
\text { nesse setor }\end{array}$ \\
\hline
\end{tabular}




\begin{tabular}{|l|l|l|}
\hline AUTORES/ANO & PERIÓDICO & TíTUTO DO TRABALHO \\
\hline $\begin{array}{l}\text { Paraíso, Soares e Almeida } \\
\text { (2009) }\end{array}$ & $\begin{array}{l}\text { Revista de Gestão Ambiental e } \\
\text { Sustentabilidade - GeAS }\end{array}$ & $\begin{array}{l}\text { Desafios e práticas para a inserção da } \\
\text { Tecnologia da Informação Verde nas empresas } \\
\text { baianas: um estudo sob a perspectiva dos } \\
\text { profissionais de tecnologia da informação }\end{array}$ \\
\hline $\begin{array}{l}\text { Nascimento Pontes e } \\
\text { Giordano (2015) }\end{array}$ & $\begin{array}{l}\text { Revista de Gestão Ambiental e } \\
\text { Sustentabilidade - GeAS }\end{array}$ & $\begin{array}{l}\text { Práticas de TI Verde em uma empresa } \\
\text { educacional para fomentar a responsabilidade } \\
\text { socioambiental }\end{array}$ \\
\hline $\begin{array}{l}\text { Schulz e Silva (2012) } \\
\text { Lunardi, Simões e Frio }\end{array}$ & $\begin{array}{l}\text { Revista de Gestão Social e } \\
\text { Ambiental }\end{array}$ & $\begin{array}{l}\text { TI Verde e eficiência energética em Data } \\
\text { Centers }\end{array}$ \\
\hline $\begin{array}{l}\text { Re14) } \\
\text { Administração }\end{array}$ & $\begin{array}{l}\text { TI Verde: uma análise dos principais } \\
\text { benefícios e práticas utilizadas pelas } \\
\text { organizações }\end{array}$ \\
\hline $\begin{array}{l}\text { Westphall e Villarreal } \\
\text { (2013) }\end{array}$ & $\begin{array}{l}\text { Revista Eletrônica de Sistemas de } \\
\text { Informação }\end{array}$ & $\begin{array}{l}\text { Princípios e tendências em Green Cloud } \\
\text { Computing }\end{array}$ \\
\hline Foñ
\end{tabular}

Fonte: Dados da pesquisa

Alguns estudos tratam da ligação entre as práticas da Tecnologia da Informação e a Sustentabilidade nas organizações. Lunardi, Simões e Frio (2014) analisam as principais práticas da TI Verde e seus respectivos benefícios. Os autores identificaram que os principais ganhos estão associados à redução de custos, à redução de insumos, ao menor consumo de energia, à melhoria da imagem institucional e à redução da emissão de gases; já dentre principais práticas mais utilizadas, destacam-se a consolidação de servidores e desktops, o uso de equipamentos mais eficientes, a reciclagem de componentes e as campanhas de conscientização.

Nesse sentido, Salles e outros autores (2016) analisam da adoção de ações da TI Verde nas organizações, examinando, mais especificamente, os motivos de adoção, as práticas implantadas, os benefícios percebidos e as dificuldades enfrentadas. Segundo os autores, as ações, em sua grande maioria, enquadram-se nas intersecções do Tripé da Sustentabilidade - ambiental, social e econômica - e por uma quarta dimensão, chamada de legal. Além disso, mostram que a implementa- ção parte, inicialmente, da identificação de benefícios financeiros e operacionais, revelando que os benefícios da dimensão ambiental surgem como uma consequência positiva das mudanças provocadas em função de aspectos econômicos e legais.

De modo complementar, o trabalho de Schulz e Silva (2012), trata dos impactos gerados pelo uso dos Data Centers no consumo de energia elétrica, sua crescente representatividade e como novas tecnologias e as melhores práticas podem ajudar neste cenário, aliando a redução do impacto ambiental com a diminuição de custos. Antes saber, os Data Centers contêm, primariamente, equipamentos eletrônicos usados para o processamento, armazenamento e comunicações de dados e são equipamentos de Tecnologia da Informação e Comunicação (TIC).

Schulz e Silva (2012) discutem, ainda, que as práticas da TI Verde devem ser consideradas na construção de novos Data Centers e na evolução deles, de modo a vir a ajudar a diminuir seu impacto ambiental, sem prejuízo a dois dos mais importantes indicadores de TIC: performance e disponibilidade, já que os Data 
Center também contêm sistemas de suporte a energia elétrica e climatização para manter alta disponibilidade, garantindo o funcionamento ininterrupto e as condições ideais de temperatura e umidade para equipamentos de TIC.

Umas das principais práticas da TI Verde é a utilização da Computação em Nuvem [(Green Cloud Computing) GCC] que se baseia nos conceitos de duas áreas: Ciência da Computação e Sustentabilidade, com o objetivo de oferecer sistemas computacionais flexíveis e eficientes. No estudo de Westphall e Villarreal (2013), a discussão gira em torno das possíveis tecnologias que viabilizam o desenvolvimento do GCC, destacando-se entre elas as tecnologias aplicadas a Hardware, aos Data Centers, Virtualização e Green Networking. Por fim, os autores elucidam que a abordagem Cloud Computing se mostra como uma excelente alternativa para utilização dos recursos computacionais, uma vez que permite consolidar a carga de trabalho de uma grande quantidade de usuários e também porque seus recursos favorecem a consolidação da carga de trabalho dentro do datacenter.

Lunardi, Lerch e Salles (2014) desenvolveram e validaram um instrumento para avaliar o grau de utilização da TI Verde. Para os autores, a mensuração do grau da TI é baseada mediante alguns critérios, expostos no Quadro 3.

Quadro 3 - Critérios de avaliação da TI Verde

\begin{tabular}{|c|c|c|}
\hline Critérios & Descrição & Autores \\
\hline $\begin{array}{l}\text { Efetividade das } \\
\text { ações sustentáveis } \\
\text { aplicadas à área de TI }\end{array}$ & $\begin{array}{l}\text { Avalia em que grau a organização implementa diferentes } \\
\text { iniciativas de modo a tornar as atividades de TI mais } \\
\text { sustentáveis, utilizando os recursos computacionais de } \\
\text { forma mais eficiente e contribuindo para o meio ambiente. }\end{array}$ & $\begin{array}{l}\text { Molla et al. (2008); } \\
\text { Murugesan (2008); } \\
\text { Brooks, Wang e Sarker } \\
\text { (2010) }\end{array}$ \\
\hline $\begin{array}{l}\text { Grau de orientação } \\
\text { ambiental e pelo } \\
\text { nível de consciência } \\
\text { socioambiental da } \\
\text { organização }\end{array}$ & $\begin{array}{l}\text { Avalia em que grau a organização está comprometida } \\
\text { com a sustentabilidade e com o suporte às inovações } \\
\text { ambientais, implementando práticas e rotinas } \\
\text { organizacionais na área de TI que encorajem o comporta- } \\
\text { mento dos funcionários a seguir as políticas de responsabi- } \\
\text { lidade ambiental que a organização apoia. }\end{array}$ & $\begin{array}{l}\text { Peloza e Hassay (2006); } \\
\text { Jenkin, Webster e } \\
\text { Mcshane (2011) }\end{array}$ \\
\hline $\begin{array}{l}\text { Monitoramento das } \\
\text { atividades de TI }\end{array}$ & $\begin{array}{l}\text { Avalia em que grau a organização gerencia as atividades e } \\
\text { medidas de TI voltadas à redução do consumo de recursos, } \\
\text { aos danos causados ao meio ambiente e aos custos } \\
\text { operacionais do negócio, além de melhorar a efetividade } \\
\text { do consumo de energia. }\end{array}$ & $\begin{array}{l}\text { Molla (2009); Melville } \\
\text { (2010); Schmidt et al. } \\
\text { (2010) }\end{array}$ \\
\hline $\begin{array}{l}\text { Pelo grau de } \\
\text { expertise ambiental } \\
\text { na área de TI }\end{array}$ & $\begin{array}{l}\text { Avalia em que grau a organização se submete a } \\
\text { experimentar, atualizar e buscar novas abordagens, } \\
\text { informações e conhecimentos referentes ao uso de } \\
\text { equipamentos e serviços para suportar as estratégias de } \\
\text { sustentabilidade ambiental na área de TI. }\end{array}$ & $\begin{array}{l}\text { Elliot e Binney (2008;) } \\
\text { Mines (2008); Kim e Ko } \\
\text { (2010) }\end{array}$ \\
\hline
\end{tabular}

Fonte: Adaptado de Lunardi, Lerch e Salles (2014). 
Para os autores, ainda é perceptível que as organizações continuam em constante pressão de clientes, concorrentes, órgãos reguladores e grupos comunitários para implementar práticas de negócio ambientalmente corretas, de modo que tentar achar um ponto de equilíbrio entre o desempenho econômico e ambiental, simultaneamente, acaba sendo uma questão estratégica.

Valença e outros autores (2017) afirmam que diante de um cenário competitivo e de crise econômica, as empresas têm buscado novas formas de gerir seus processos e operações de modo eficaz e sustentável. Nesse sentido Radons, Battistella e Grohmann (2012), identificam se a inovação verde influencia positivamente a vantagem competitiva das empresas. Para os autores, existe uma preocupação com a questão ambiental, tanto no desenvolvimento de produtos como no processo de operação e que as empresas estão voltadas para a busca de vantagem competitiva por meio de práticas ambientais responsáveis. Porém, alinhado a isso, deve-se concentrar seus esforços para potencializar a inovação no processo verde, que é percebida como mais influente para alcance da vantagem competitiva.

Para Paraíso, Soares e Almeida (2009), as evoluções tecnológicas oferecem maior capacidade de processamento de dados por um custo mais baixo, porém a resistência para a adoção da TI Verde é formada pelo valor do investimento de adoção da TI, com isso umas das maiores dificuldades encontradas pelas empresas para adotarem a TI Verde é o alto custo para realizar o processo de troca do parque tecnológico, sendo que cerca de $70 \%$ das empresas optam por não fazer.

Porém, Nascimento Pontes e Giordano (2015) afirmam que os colaboradores de TI podem servir como influenciadores e auxiliar os gestores na tomada de decisão no gerenciamento de seus ecossistemas tecnológicos menos impactantes possíveis ao meio ambiente. De modo que, o fator educacional possa ser utilizado para analisar os itens avaliados para implementar estratégias de contribuição com a responsabilidade socioambiental e com o desenvolvimento sustentável.

\section{CONSIDERAÇÕES FINAIS}

Como considerações finais pode-se afirmar que há pesquisas científicas a respeito do tema, porém, em quantidade extremamente inferior à necessidade atual. A busca pela sustentabilidade ambiental no uso da Tecnologia da Informação é tema recorrente na sociedade vigente, vem sendo fortemente debatido, porém, os esforços para atender esta demanda ainda são escassos. 0 fato impulsionador está mais ligado à redução de custos, atender questões legais e ao chamar atenção do cliente do que o cuidado com o meio ambiente em si.

As empresas, em sua maioria, detém atenção para aspectos relacionados à redução de custos, à redução de insumos, ao menor consumo de energia, à melhoria da imagem institucional e à redução da emissão de gases. Quanto às práticas mais utilizadas, destacam-se a consolidação de servidores e desktops, o uso de equipamentos mais eficientes, a reciclagem de componentes e as campanhas de conscientização.

Especificamente à TI Verde, a principal prática é a utilização da Computação em Nuvem Green Cloud Computing (GCC) que se baseia nos conceitos de duas áreas: Ciência da Computação e Sustentabilidade, com o objetivo de oferecer sistemas computacionais flexíveis e eficientes, destacando-se entre elas as tecnologias aplicadas a Hardware, aos Data Centers, Virtualização e Green Networking. Os autores elucidam que a abordagem Cloud Computing se mostra como uma excelente alternativa para utilização dos recursos computacionais, uma vez que permite consolidar a carga de trabalho de uma grande quantidade de usuários e também porque seus recursos favorecem a consolidação da carga de trabalho dentro do datacenter.

Para estudos futuros, sugere-se que sejam realizadas buscas mais aprofundadas em artigos nacionais e internacionais, de modo a identificar novos fatores de imersão nos estudos voltados as práticas da Tecnologia a de Informação Verde. Bem como, aprimorar as ferramentas existentes e promover um conhecimento voltado para a tomada de decisões gerenciais a respeito da adoção de TI Verde. 


\section{REFERÊNCIAS}

BOZZELLI, Paolo; GU, Qing; LAGO, Patricia. A systematic literature review on green software metrics. VU University, Amsterdam, 2013.

BROOKS, Stoney; WANG, Xuequn; SARKER, Saonee. Unpacking Green IT: A review of the existing literature. 2010.

BUENAFUENTE, S.M.F. et al. Sustentabilidade corporativa e o profissional de secretariado executivo. XVII CONSEC - 26 A 29/05/2010 FORTALEZA/CE, 2010.

CASTRO, Bruno Roberto Viana et al. O marketing verde na tecnologia da informação: percepções das atitudes e comportamentos dos profissionais de TI e ações para incrementar o marketing verde nesse setor. Revista de Gestão Ambiental e Sustentabilidade, v.5, n.1, p.45, 2016.

DANIEL, Rafael Henrique Martins Antonio; AGUIAR, Kátia Cristina Alberto Aguiar. Sustentabilidade. 0 ciclo do sucesso: responsabilidade social, econômica e ambiental como vantagem competitiva. Revista Ciências Gerenciais, v.18, n.28, p.114-120, 2014.

DIEL, Fábio José et al. Investimentos ambientais e desempenho econômico financeiro. REUNA, v.19, n.2, p.113-134.

DOLCI, Decio Bittencourt et al. Implementation of green IT in organizations: A structurational view. Revista de Administração de Empresas, v.55, n.5, p.486-497, 2015.

ELKINGTON, John. Towards the sustainable corporation: win-win-win business strategies for sustainable development. California Management Review, v.36, n.2, p.90-100, 1994.

ELLIOT, Steve; BINNEY, Derek. Environmentally sustainable ICT: Developing corporate capabilities and an industry-relevant IS research agenda. PACIS 2008 Proceedings, p. 209, 2008.

HEPPER, Eduardo Luís; HANSEN, Peter Bent; SANTOS, Jane Lucia S. Iniciativas sustentáveis e desempenho organizacional: uma análise das publicações na base web of science. Revista de Gestão Ambiental e Sustentabilidade, v.5, n.2, p.98, 2016.

JENKIN, Tracy A.; WEBSTER, Jane; MCSHANE, Lindsay. An agenda for 'Green'information technology and systems research. Information and Organization, v.21, n.1, p.17-40, 2011.

KIM, Yong Seog; KO, Myung. Identifying Green IT Leaders with Financial and Environmental Performance Indicators. AMCIS, 2010. p.54.

LUNARDI, Guilherme Lerch; ALVES, Ana Paula Ferreira; SALLES, Ana Carolina. Desenvolvimento de uma escala para avaliar o grau de utilização da tecnologia da informação verde pelas organizações. 2014.

LUNARDI, Guilherme Lerch; SIMÕES, Renata; FRIO, Ricardo Saraiva. TI verde: uma análise dos principais benefícios e práticas utilizadas pelas organizações. REAd-Revista Eletrônica de Administração, v.20, n.1, 2014.

MARCONDES, A.W.; BACARJI, C.D. ISE: sustentabilidade nos mercados de capitais. São Paulo: Report, 2012.

MELVILLE, Nigel P. Information systems innovation for environmental sustainability. MIS quarterly, v.34, n.1, p.1-21, 2010.

MINES, Christopher. The dawn of green IT services. Cambridge-MA: Forrester Research, 2008.

MOLLA, Alemayehu et al. E-readiness to G-readiness: Developing a green information technology readiness framework. ACIS 2008 Proceedings, p.35, 2008. 
MOLLA, Alemayehu. Organizational motivations for Green IT: Exploring Green IT matrix and motivation models. PACIS 2009 Proceedings, p.13, 2009.

MURUGESAN, San. Harnessing green IT: Principles and practices. IT professional, v.10, n.1, 2008.

MURUGESAN, San. Making IT green. IT professional, v.12, n.2, p.4-5, 2010.

NASCIMENTO PONTES, Floriana; GIORDANO, Fabio. Práticas de TI Verde em uma empresa educacional para fomentar a responsabilidade socioambiental.

Revista de Gestão Ambiental e Sustentabilidade, v.4, n.2, 2015.

OLIVEIRA, Rodrigo Cesar Reis de; SANTOS, Ernani Marques; GONZALEZ JÚNIOR, Ivo Pedro. Uma proposta para análise da adoção de tecnologias da informação em micro e pequenas empresas a partir da adaptação do modelo TOE (Technology, Organization and Environment). Revista

Brasileira de Administração Científica, v.4, n.2, p.257-272, 2013.

PAIVA, P.R. Contabilidade ambiental: evidenciação dos gastos ambientais com transparência e focada na prevenção. São Paulo: Atlas, 2003.

PARAÍSO, Marcos Ramos de Almeida; SOARES, Thereza Olívia Rodrigues; ALMEIDA, Leandro Antonio de. Desafios e Práticas para a Inserção da Tecnologia da Informação Verde nas Empresas Baianas: um estudo sob a perspectiva dos profissionais de Tecnologia da Informação 10.5773/rgsa. v3i3. 178. Revista de Gestão Social e Ambiental, v.3, n.3, 2009.

PEÇANHA, Reynaldo Schirmer; IIZUKA, Edson Sadao. Análise da produção científica brasileira sobre sustentabilidade entre os anos de 2008 a 2011.

Revista de Gestão Ambiental e SustentabilidadeGeAS, v.3, n.1, p.1-17, 2014.
PELOZA, John; HASSAY, Derek N. Intraorganizational volunteerism: Good soldiers, good deeds and good politics. Journal of Business Ethics, v.64, n.4, p.357-379, 2006.

PINHEIRO, Sara Maria Gomes et al. Implementation of environmental management tools to support the management of solid waste in the municipality of Rio Tinto/PB. Revista Geama, v.6, n.1, p.160-169, 2016.

PUPPIM, J.A.O. Uma avaliação dos balanços sociais das 500 maiores. RAE Eletrônica, v.4, n.1, 2005.

RADONS, Daiane Lindner; BATTISTELLA, Luciana Flores; GROHMANN, Márcia Zampieri. Vantagem competitiva das empresas: qual a influência da inovação verde? Revista Brasileira de Administração Científica, v.3, n.3, p.97-111, 2013.

SALLES, Ana Carolina et al. Tecnologia da informação verde: um estudo sobre sua adoção nas organizações. RAC-Revista de Administração Contemporânea, v.20, n.1, 2016.

SCHMIDT, Nils-Holger et al. Predictors of Green IT Adoption: Implications from an Empirical Investigation. AMCIS, p.367, 2010.

SCHULZ, Murilo Alexandre; SILVA, Tania Nunes. TI verde e eficiência energética em data centers. Revista de Gestão Social e Ambiental, v.6, n.2, p.121-133, 2012.

SOUZA, Taciana Rodrigues de; BRIGHENTI, Josiane; HEIN, Nelson. Investimentos ambientais e desempenho econômico-financeiro das empresas brasileiras listadas no índice de sustentabilidade empresarial-ISE. REUNA, v.21, n.2, p.97-114, 2016.

VALENÇA, Antonio Karlos Araújo et al. Brazilian Scientific Production on IT adoption in Micro and 
Small Enterprises. 14th International conference on information systems \& technology management CONTECSI, 14. 2017, São Paulo. Proceedings... São Paulo: FEA/USP, 2017.
WESTPHALL, Carlos Becker; VILLARREAL, Sergio Roberto. Princípios e tendências em green cloud computing. Revista Eletrônica de Sistemas de Informação, v.12, n.1, p.1-19, 2013.
Recebido em: 3 de Setembro de 2017 Avaliado em: 14 de Setembro de 2017 Aceito em: 12 de Outubro de 2017
1 Possui graduação em Engenharia de Produção pela Faculdade de Administração e Negócios de Sergipe (FANESE). Mestrando em Engenharia de Materiais pela Universidade Federal de Sergipe (UFS). E-mail: akavalenca@gmail.com

\begin{abstract}
2 Doutor em Administração pelo Núcleo de Pós-Graduação em Administração da UFBA (NPGA-UFBA). Mestre em Administração pelo Programa de Pós-graduação em Administração da UFPE (PROPAD-UFPE). Graduado em Administração pela Universidade Federal da Paraíba. Professor de graduação e pós-graduação. Atuou como coordenador do curso de bacharelado em Administração da Faculdade de Administração e Negócios de Sergipe FANESE. E-mail: rodrigopesquisando@gmail.com

3 Doutor em Administração (2017) e Mestre em Administração (2012) pela Universidade Federal da Bahia (UFBA), tem Especialização em Redes de Computadores (2001) pela Faculdade Ruy Barbosa (FRB) e Graduação em Processamento de Dados (1999) também pela FRB. É Tecnologista do Centro de Pesquisas Gonçalo Moniz (CPqGM) da Fundação Oswaldo Cruz (FIOCRUZ). E-mail: aealbuquerque@gmail.com
\end{abstract}

4 Graduanda de bacharelado em Administração na Universidade Federal Rural de Pernambuco. E-mail: viviaanfariaas@gmail.com 